\title{
USDA revises policies on licensing of facilities and IACUC membership
}

On 7 March 2006, the United States Department of Agriculture, Animal and Plant Health Inspection Service, Animal Care (USDA, APHIS, AC) revised two of its Animal Care policies. Policy \# 10, which relates to licensing and registration of producers of animal-derived products, and Policy \#15, which deals with membership of the IACUC, are two of the 29 policies that comprise the USDA Animal and Plant Health Inspection Animal Care Policy Manual ${ }^{1}$.

\section{USDA Policy \#10}

USDA revised Policy \#10 to clarify the requirements for licensing and registration of producers of antibodies, sera, or other animal parts, producers of genetically engineered and cloned animals, and licensed exhibitors.

Any facility that produces antibodies or antisera must be registered as a research facility since it must test animals for their immune response, and select animals to produce antibodies. A research facility selling antibodies, antisera, or other body parts for research, teaching, or testing must have a dealer's license in addition to its registration. A research facility that only produces antibodies or antisera under contract with a specific investigator does not require a license.

Producers of genetically engineered and cloned animals must be registered as research facility. A facility that clones USDA-regulated animals is considered to be breeder, and must be licensed as a dealer.

Licensed exhibitors who conduct invasive, painful, or stressful procedures in biomedical research for nonhusbandry purposes, or research on dogs or cats must be registered. Registration is not required, if collection methods are performed as an adjunct to normal husbandry or veterinary procedures.

\section{USDA Policy \#15}

USDA revised Policy \#15 to clarify the individual roles of IACUC members. According to the revised policy, IACUC members, including nonaffiliated members, which had been required to have access to training, must now be provided with training, to better assess the facilities and their programs of animal care.

The revised Policy \#15 discourages, but does not prohibit, the assignment of one person to more than one role on the IACUC to avoid conflicts of interest and to prevent one person from having too much influence. The revised policy also specifies that an IACUC member cannot be involved in the review of his or her own animal care and use protocol.

1. USDA APHIS AC. USDA Animal and Plant Health Inspection Animal Care Policy Manual. http:// www.aphis.usda.gov/ac/polmanpdf.html.

\section{British scientists to report severity of animal procedures retrospectively}

In December 2005, the UK-based Laboratory Animal Science Association (LASA), and the Animal Procedures Committee (APC), completed a pilot study to "assess the feasibility of collecting and reporting data on the sesverity of adverse effects caused to animals used in procedures regulated under the Animals (Scientific Procedures) Act 1986 (ref. 1)."

If the group's recommendations are implemented, UK investigators will be required to provide retrospective reports detailing the severity of pain and distress experienced by their laboratory animals. This information could be published in annual reports and used to refine future experiments.

The working group developed an intensity-duration severity grid, in which the two parameters are considered independently. They found that the grid system "works well with procedures that have relatively simple severity profiles but again is less successful in providing a meaningful reflection of severity in more complex, longer-term procedures ${ }^{1}$." Therefore, they went on to propose the use of a two-grid system that describes both the maximum severity and the severity over the remainder of the procedure.
The working group is proposing to extend their work to accomplish the following:

- "[C] onsider simplification of the proposed reporting scheme;

- "[A] ssess its appropriateness across a wider range of establishments;

- "[E]valuate the consistency of response to the scoring system;

- "[I]dentify a process for developing a glossary of severity codes for a range of protocols and outcomes;

- "[C]larify a number of unresolved issues in the application of the coding scheme;

- "[C] onduct a detailed impact assessment; and

- "[A] ssess the value of the scheme in generating data that can assist public understanding of the severity of effects caused to animals used in regulated scientific procedures ${ }^{1}$."

1. Smith, D. et al. Reporting the severity of animal procedures retrospectively. Report of a LASA/APC Pilot Study to assess the feasibility of collecting and reporting data on the severity of adverse effects caused to animals used in procedures regulated under the Animals (Scientific Procedures) Act 1986 (December 2005). http:// www.apc.gov.uk/reference/lasa-report.pdf. 